\title{
(S)TEM Characterization of Chemically Exfoliated Black Phosphorus
}

Amy Ng${ }^{1}$, Thomas E. Sutto ${ }^{2}$, Yexin Deng ${ }^{3}$, Rhonda M. Stroud ${ }^{2}$, Todd H. Brintlinger ${ }^{2}$, Peide D. Ye ${ }^{3}$, Nabil D. Bassim²

1. NRC Postdoctoral Associate, Naval Research Laboratory, Washington, DC, USA.

2. Naval Research Laboratory, Materials Science \& Technology Division, Washington, DC, USA.

3. Purdue University, School of Electrical and Computer Engineering, West Lafayette, IN, USA.

Phosphorene is the 2-dimensional form of black phosphorus and a close relative of graphene. It has a nonzero fundamental band gap that gives rise to semiconductor properties, which makes it highly desirable for numerous applications in optoelectronics [1] and as a replacement channel for conventional semiconductor devices [2]. However, difficulties in isolating large area single-, few-, or multi-layer sheets are an impediment to realizing the aforementioned applications. We are investigating multiple routes for optimal production of phosphorene sheets via chemical intercalation combined with mechanical agitation. Utilizing solvent systems of increasing viscosity, i.e. chloroform ( $0.57 \mathrm{cP})$, ethanol (1.1 cP), choline chloride-urea/ethanol (2.84 cP), and EMI-BF4 (66 cP), we have obtained flakes of differing thickness and sizes. Following our solvent treatments to obtain micro-scale flakes, we characterize the structure and composition with both high-resolution and aberration-corrected scanning transmission electron microscopy (HRTEM and ac-STEM, a JEOL JEM2200FS and Nion UltraSTEMX 200 at the U.S. Naval Research Laboratory, at $200 \mathrm{kV}$ and $60 \mathrm{kV}$, respectively), as seen in Fig. 1. Flake size, quality, and quantity as a function of the solvent system will be presented.

In our process, a rough mechanically exfoliated black phosphorus piece was submerged in a specific solvent, and then sonicated in a water bath for 10 minutes at $20 \mathrm{~W}$. The resultant colloid was dropcast onto a lacy carbon TEM grid, and then rinsed with a solvent that solubilizes the excess ionic liquid, if present. Finally, the grid is immersed in a large excess of a rinsing solvent (ethanol/chloroform) for 1 to 2 days with gentle agitation. Samples were characterized using electron energy loss spectroscopy (EELS), energy dispersive spectroscopy (EDS), and electron diffraction.

The quantity of phosphorene flakes decreased between solvent systems with increasing solution viscosity. Using a constant power input via sonication $(20 \mathrm{~W})$, each solvent produced flakes/stacks of sheets of differing quantities, where the least viscous solutions had a greater yield of flakes (chloroform, ethanol), while the highly viscous ionic liquids did not exfoliate as many (choline chloride urea). The lateral dimensions of the flakes (at 2-3 $\mu \mathrm{m}$ on a side) are, overall, comparable to higher powered sonication as shown elsewhere [3]. Although single-layer sheets were not measured, multiple layered stacks down to 2-3 layers (as measured by HAADF image intensity) were obtained for all solvent systems. Due to the difficulty of removing all ionic liquid from the flakes, sheets that are suspended in solution are unlikely to preferentially adhere to the grid before it becomes rinsed off. Additionally, ionic liquids, having a more viscous consistency, absorb more of the ultrasonic energy generated by the cup sonicator, thus lowering the possibility of exfoliating the bulk piece of black phosphorus.

Our conclusion based on these results is that solvents, in addition to the ionic liquids, play a role in the exfoliation of black phosphorus. Additionally, we are evaluating the role of sonication power on the quality and quantity of flakes, similar to other studies [3, 4]. We continue to explore the relationship between bulk materials, chemical intercalation and mechanical agitation in these 2-D materials. 


\section{References:}

[1] H Liu, et al, ACS Nano 8 (2014), p. 4033-4041.

[2] Y Xu, J Dai, and XC Zeng, J Phys Chem Lett 6 (2015), p. 1996-2002.

[3] W Zhao, et al, ACS Appl Mater Interfaces (2015).

[4] D Hanlon, et al, Nat Comm (2015).

[5] The authors acknowledge funding from the Office of Naval Research (Naval Research Laboratory Basic Research Program).
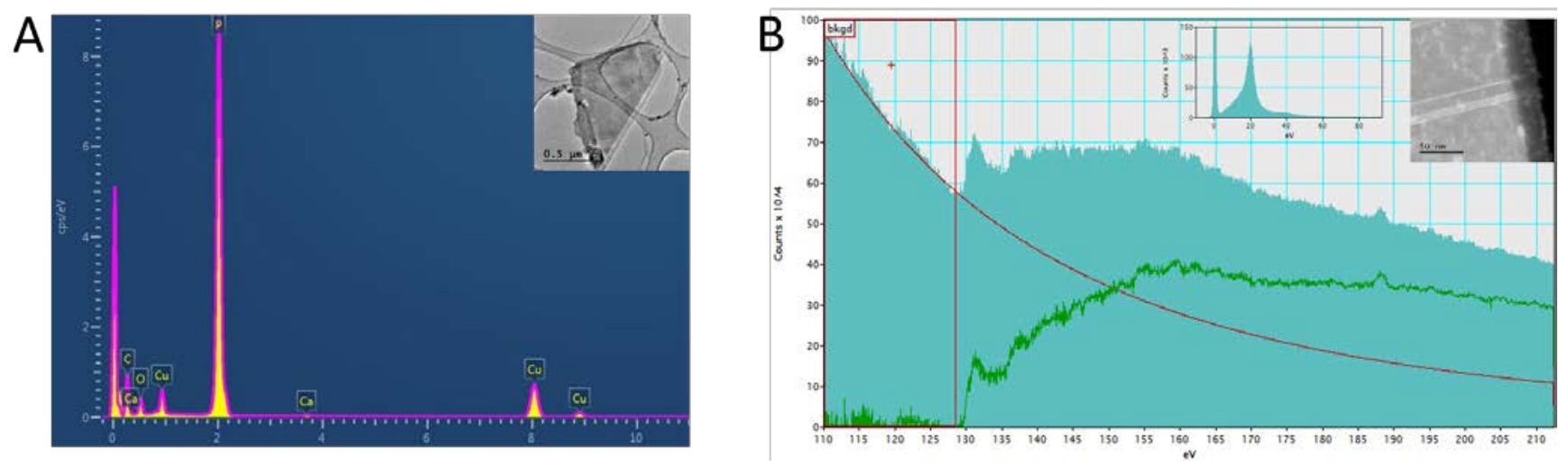

Figure 1. (A) EDS spectrum of a representative phosphorene flake (inset) showing minimal oxidation of the phosphorene sheet. (B) Core-loss EELS of a flake (inset, top right) and low loss EELS spectra.

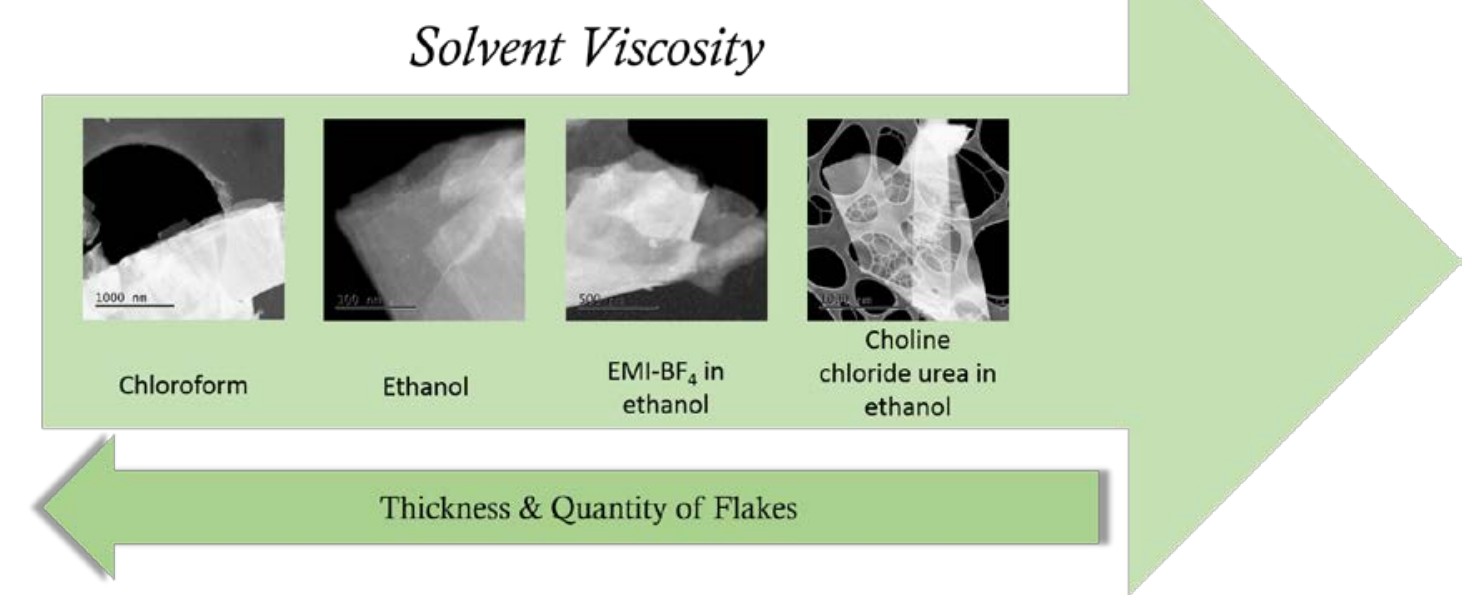

Figure 2. As a function of solvent system viscosity, the produced flakes after sonication decrease in thickness and quantity. 